\title{
Quantification of apixaban in human plasma using ultra performance liquid chromatography coupled with tandem mass spectrometry
}

\author{
Hyeon-Cheol Jeong ${ }^{1}$, Tae-Eun Kim ${ }^{2}$ and Kwang-Hee Shin ${ }^{1 *}$ \\ ${ }^{1}$ College of Pharmacy, Research Institute of Pharmaceutical Sciences, Kyungpook National University, Daegu 41566, Korea \\ ${ }^{2}$ Department of Clinical Pharmacology, Konkuk University Medical Center, Seoul 05030, Republic of Korea \\ ${ }^{\star}$ Correspondence: K. H. Shin; Tel: +82-53-950-8582, Fax: +82-53-950-8557, E-mail: kshin@knu.ac.kr
}

\section{Check for updates}

Received 13 Feb 2019

Revised 11 Mar 2019

Accepted 11 Mar 2019

\section{Keywords}

Anticoagulant,

Apixaban,

Bioanalytical method,

UPLC-MS/MS

pISSN: 2289-0882

elSSN: 2383-5427
Apixaban, an inhibitor of direct factor $\mathrm{Xa}$, is used for the treatment of venous thromboembolic events or prevention of stroke. Unlike many other anticoagulant agents, it does not need periodic monitoring. However, monitoring is still required to determine the risk of bleeding due to overdose or surgery. Usually, apixaban concentrations are indirectly quantified using an anti-factor Xa assay. However, this method has a relatively narrow analytical concentration range, poor selectivity, and requires an external calibrator. Therefore, the goal of current study was to establish an analytical method for determining plasma levels of apixaban using ultra performance liquid chromatographytandem mass spectrometry (UPLC-MS/MS). To this end, apixaban was separated using $2.5 \mathrm{mM}$ ammonium formate ( $\mathrm{pH} 3.0$ ) (A) and $100 \%$ methanol containing $0.1 \%$ formic acid (B) using the gradient method with a Thermo hypersil GOLD column. The mass detector condition was optimized using the electrospray ionization (ESI) positive mode for apixaban quantification. The developed method showed sufficient linearity (coefficient of determination $\left[r^{2} \geq 0.997\right]$ ) at calibration curve ranges. The percentage (\%) changes in accuracy, precision, and all stability tests were within $15 \%$ of the nominal concentration. Apixaban concentration in plasma from healthy volunteers was quantified using the developed method. The mean maximum plasma concentration $\left(\mathrm{C}_{\max }\right)$ was $371.57 \mathrm{ng} / \mathrm{mL}$, and the median time to achieve the $\mathrm{C}_{\max }\left(\mathrm{T}_{\max }\right)$ was $4 \mathrm{~h}$ after administration of $10 \mathrm{mg}$ apixaban alone. Although the results showed low extraction efficiency $(\sim 16 \%)$, the reproducibility (\% change was within $15 \%$ of nominal concentration) was reliable. Therefore, the developed method could be used for clinical pharmacokinetic studies.

\section{Introduction}

The risk of venous thromboembolism increases in patients post-surgically, such as after hip or knee replacement, fracture surgery, or atrial fibrillation.[1,2] Generally, vitamin K antagonists such as warfarin, phenprocoumon, and acenocoumarol are used to prevent thromboembolism risk,[3] but they have

Copyright @ 2019 Translational and Clinical Pharmacology

(a) It is identical to the Creative Commons Attribution Non-Commercial License (http://creativecommons.org/licenses/by-nc/3.0/)

(2) This paper meets the requirement of KS X ISO 9706, ISO 9706-1994 and ANSI/NISO Z.39.48-1992 (Permanence of Paper).

Reviewer

This article reviewed by peer experts who are not TCP editors. some disadvantages. First, international normalized ratio (INR) monitoring is commonly required for patients on vitamin $\mathrm{K}$ antagonists because of their narrow therapeutic index.[4,5] Second, vitamin $\mathrm{K}$ antagonists have long half-lives of approximately $40 \mathrm{~h}$, so anticoagulant effects remain after treatment has been discontinued.[6] Finally, vitamin K antagonists have high inter-individual variabilities according to the cytochrome P450 2C9 (CYP2C9) and vitamin K epoxide reductase 1 (VKORC1) enzyme genotypes.[7-9] Furthermore, dietary control is recommended such as limiting vitamin K-rich foods.[10] Novel oral anticoagulants (NOACs) such as apixaban, rivaroxaban, edoxaban, and dabigatran have been developed to overcome these disadvantages.[11] NOACs have shorter half-lives (ap- 
proximately $8-15,5-13,6-11$, and $12-14 \mathrm{~h}$ for apixaban, rivaroxaban, edoxaban, and dabigatran, respectively) than that of warfarin (approximately $40 \mathrm{~h}$ ), so their anticoagulant effects are easily controlled.[4,12,13] Furthermore, dietary limitations are not required for these drugs. [14,15]

Unlike conventional anticoagulants, apixaban does not require periodic monitoring, however, it does require monitor under certain situations such as following the administration of an overdose or before surgery.[6] Generally, the chromogenic anti-Xa assay is used for monitoring apixaban concentrations clinically.[16] Specific external calibrators are required to quantify apixaban or rivaroxaban because this method was originally designed to quantify plasma heparin concentrations. [17] In addition, the sensitivities of the chromogenic assay and dilute Russell viper venom time (DRVV-T) method were 10-25 and 100-200 ng/mL, respectively.[18,19] Furthermore, these methods have poor selectivity for apixaban quantification. For example, in a previous study, apixaban was even detected in the control group that was not administered an anticoagulant.[20] Therefore, more sensitive and selective analytical methods for apixaban are required for pharmacokinetic studies and effective drug concentration monitoring.

Liquid chromatography-mass spectrometry (LC-MS) is generally used for analysis in various fields and is mainly used to quantify drug concentrations clinically. Once the method is established, analytes can be rapidly analyzed daily and it has the advantage of high sensitivity.[21-23] Recently, published articles suggest that analytical methods using LC-MS can be used to quantify not only apixaban but also other NOACs such as rivaroxaban simultaneously.[21,24] The objective of current study was to establish a quantification method for detecting apixaban in human plasma using UPLC-MS/MS and to apply the method to a clinical studies.

\section{Methods}

\section{Reagents}

Apixaban and apixaban-d3 (internal standard, IS) were obtained from Toronto Research Chemicals (Ontario, Canada). Methanol, ammonium formate, and formic acid were obtained from Sigma-Aldrich (St. Louis, MO, USA) and Duksan (Korea). All reagents used in this study were either LC grade or extrapure grade. Millipore Milli-Q system at 18.2 M $\Omega$ (Billerica, MA, USA) was used to obtain deionized water. Drug-free blank plasma containing ethylenediaminetetraacetic acid (EDTA)-K3 was bought from Biochemed (Winchester, VA, USA).

\section{Chromatographic methods}

The analysis was performed on the Acquity ${ }^{\mathrm{TM}}$ UPLC (Waters, MA, USA) and Xevo TQ-MS Triple Quadrupole Tandem Mass Spectrometer (Waters, MA, USA). The separation of apixaban in plasma was conducted using a mobile phase consisting of 2.5 $\mathrm{mM}$ ammonium formate (pH 3.0) (A) and 100\% methanol con- taining $0.1 \%$ formic acid (B) on the Thermo Hypersil Gold C18 column $(150 \times 2.1 \mathrm{~mm}, 1.9 \mu \mathrm{m})$. The run time using the gradient method was $3 \mathrm{~min}$. Gradient method was applied as follows: 0-0.2 $\min (45 \% \mathrm{~A}), 0.2-0.5 \min (15 \% \mathrm{~A}), 0.5-2.0 \mathrm{~min}(15 \% \mathrm{~A})$, 2.0-2.1 $\mathrm{min}(45 \% \mathrm{~A})$, and $2.1-3.0 \mathrm{~min}(45 \% \mathrm{~A})$. The flow rate was $0.35 \mathrm{~mL} / \mathrm{min}$. The column oven and autosampler temperatures were set to $40^{\circ} \mathrm{C}$ and $10^{\circ} \mathrm{C}$, respectively, and maintained during analysis. Mass detection was conducted on positive ionization using multiple reaction monitoring (MRM). Mass transitions were set to $\mathrm{m} / \mathrm{z} 460.17 \rightarrow 199.09$ for apixaban and $\mathrm{m} /$ $\mathrm{z} 463.13 \rightarrow 202.09$ for the IS. The analyzed data were handled by Masslynx version 4.1 (Waters, MA, USA).

\section{Stock and working solutions}

Apixaban and the IS stock solution $(1 \mathrm{mg} / \mathrm{mL})$ were dissolved in 100\% methanol. All of the calibration curve and quality control (QC) working solutions for apixaban were prepared by dilution using methanol. Calibration curve working solutions were prepared to $10,20,50,100,200,500,1000$, and $5000 \mathrm{ng} / \mathrm{mL}$. Working solutions for low, medium, high, and dilution integrity QC (LoQC, MeQC, HiQC, and DiQC, respectively) samples were prepared to $20,150,4,000$, and $10,000 \mathrm{ng} / \mathrm{mL}$, respectively. The IS was diluted to $1 \mu \mathrm{g} / \mathrm{mL}$.

\section{Sample preparation}

The plasma completely thawed at ambient temperature (18$21^{\circ} \mathrm{C}$ ) and was then briefly vortexed. Apixaban was extracted by protein precipitation (PP) from the plasma, and then $100 \mu \mathrm{L}$ sample was transferred into a microcentrifuge tube to which $50 \mu \mathrm{L}$ IS (apixaban-d3, $1 \mu \mathrm{g} / \mathrm{mL}$ in $100 \%$ methanol) was added. Then $450 \mu \mathrm{L}$ of $100 \%$ methanol (3-fold volume of the sample) was added to the sample, followed by gentle vortexing for $5 \mathrm{~min}$ and centrifugation for $10 \mathrm{~min}$ at $13,000 \mathrm{rpm}$. The methanol phase was transferred into a vial for subsequent analysis.

\section{Validation}

Validation of the analytical method was performed in accordance to "Guidance for Industry Bioanalytical Method Validation" published by the US Food and Drug Administration and "Guideline on Bioanalytical Method Validation" published by the Korean Ministry of Food and Drug Safety (MFDS).[25,26]

\section{Accuracy and precision}

To measure accuracy and precision, lower limit of quantification (LLOQ), LoQC, MeQC, and HiQC samples were analyzed five times within each batch (intra-batch), and consecutively analyzed for three independent batches (inter-batch). The acceptance criteria of accuracy and precision were within $\pm 20 \%$ for the LLOQ and $\pm 15 \%$ for the LoQC, MeQC, and HiQC.

\section{Linearity and sensitivity}

Calibration standards for apixaban were made by spiking the working solution into drug-free blank plasma. The calibration 
standards included a double blank (no apixaban or IS), blank (only IS), and eight-point samples (both apixaban and IS). Linearity was evaluated at a concentration range of $1-500 \mathrm{ng} / \mathrm{mL}$. The coefficient of correlation ( $\mathrm{r}$ ) of linear regression was used to determine if this value was greater than 0.997 .

\section{Carry-over and matrix effect}

Carry-over was performed to determine if a high concentration of sample would affect the analysis of the next sample. A volume of $1,000 \mathrm{ng} / \mathrm{mL}$ sample (upper limit of quantification, ULOQ) was injected prior to injection of the double blank sample five times. When injecting the blank sample, the peak areas of apixaban and IS were less than $20 \%$ and $5 \%$ of LLOQ, respectively. The matrix effects were performed to assess the effects of the endogenous compounds on the analysis. LoQC and HiQC samples were prepared using six different individual plasma. The matrix effect calculated the peak area ratios for the analyte and IS of six independent LoQC and HiQC samples, and evaluated whether the $\mathrm{CV}(\%)$ was within $15 \%$.

\section{Extraction efficiency and dilution integrity}

The assessment of extraction efficiency was performed by comparing the apixaban area ratios of samples added before and after extraction. To assess the impact of dilution on analyte quantification, the DiQC sample was diluted 1:10 and 1:50, and then the diluted samples were analyzed. The apixaban concentration in the diluted sample was back-calculated by multiplying the result by the dilution factor.

\section{Stability}

Sample stability was assessed by analyzing post-processed samples that were stored in an autosampler at $10^{\circ} \mathrm{C}$ overnight. Short-term working solution and stock solution stability were assessed after the sample was kept at laboratory temperatures $\left(18-21^{\circ} \mathrm{C}\right)$ for $10 \mathrm{~h}$. We compared the apixaban peak area ratio of the sample left on the table with the newly prepared sample to obtain short-term stability. The freeze-thaw stability was measured by analyzing the apixaban concentration after repeating three cycles of freeze-thaw at $-80^{\circ} \mathrm{C}$ and laboratory temperature for the LoQC and HiQC samples. Reproducibility of re-injection was assessed by comparing the measured concentrations of the LoQC and HiQC samples analyzed in the previous batch with the freshly prepared QC samples. The acceptance criteria of the stability were that the percentage (\%) change of the calculated concentration should be within $15 \%$ of the theoretical concentration.

\section{Clinical study design}

The clinical study was conducted at Clinical Trial Center of Konkuk University Medical Center (Seoul, South Korea) in 23 subjects who voluntarily agreed to participate. Approval of the clinical study was obtained by the Institutional Review Board of Konkuk University Medical Center (IRB No. KUH1280105).
In the first period, the 23 subjects were administered a $10 \mathrm{mg}$ apixaban tablet (Eliquis, BMS Pharmaceutical Korea Ltd., Seoul, South Korea) alone, followed by a wash-out period. In the second period, 16 patients were administered $10 \mathrm{mg}$ apixaban with $20 \mathrm{mg}$ rosuvastatin (Crestor Tab., AstraZeneca, Seoul, South Korea) and 7 were administered $10 \mathrm{mg}$ apixaban and $250 \mathrm{mg}$ lapatinib tablets (Tykerb Tab., Novartis Korea, Seoul, South Korea). To quantify apixaban, whole blood was collected at predose $(0 \mathrm{~h})$ and $1,2,3,4,6,8,11$, and $24 \mathrm{~h}$ after administration in EDTA- $\mathrm{K}_{3}$ tube. The plasma was isolated by centrifugation of the collected blood at 3,000 rpm for $10 \mathrm{~min}$ at $4^{\circ} \mathrm{C}$ and transferred into microcentrifuge tubes. The plasma was stored at $-70^{\circ} \mathrm{C}$ until subsequent analysis.

\section{Results}

\section{Method development}

The mass spectra and chemical structures of apixaban and IS are presented in Figure 1. In the electrospray ionization (ESI) positive mode, apixaban and IS showed good responses. Isocratic and gradient methods were tested for sample separation using deionized water, $0.1 \%$ formic acid, ammonium formate solution, $100 \%$ methanol, $100 \%$ methanol containing $0.1 \%$ formic acid, $100 \%$ acetonitrile, and $100 \%$ acetonitrile containing $0.1 \%$ formic acid. The results showed that the gradient method was more appropriate than the isocratic method. The separation method was developed based on a previous report,[21] and modified with $2.5 \mathrm{mM}$ ammonium formate ( $\mathrm{pH} 3.0$ ) (a) and methanol containing $0.1 \%$ formic acid (b).

\section{Method validation}

\section{Accuracy and precision}

The accuracy ranged from $94.8 \%$ to $111.4 \%$ and from $98.8 \%$ to $106.2 \%$ for the intra-batch and inter-batch, respectively. The precision range was $0.49-4.54 \%$ and $2.24-8.72 \%$ for the intrabatch and inter-batch, respectively. The accuracies and precisions of the inter-batch and intra-batch are shown in Table 1.

\section{Linearity and sensitivity}

The calibration curve with a weighting factor of $1 / \mathrm{x}^{2}$ showed good linearity ranging from 1 to $500 \mathrm{ng} / \mathrm{mL}\left(\mathrm{r} \geq 0.998\right.$ and $\mathrm{r}^{2}$ $\geq 0.997)$. In the LLOQ ( $1 \mathrm{ng} / \mathrm{mL})$, signal-to-noise was $>10$. The double blank sample and chromatogram of the LLOQ are shown in Figure 2 and 3.

\section{Carry-over and matrix effect}

To determine the carry-over effect, the ULOQ sample (500 $\mathrm{ng} / \mathrm{mL}$ ) was injected and then five double blank samples were injected. The results indicated that there was no interference after injecting the double blank sample. After injecting the LoQC and HiQC samples prepared from six different individual plasma samples, the percentage coefficient of variation (\% CV) 
A
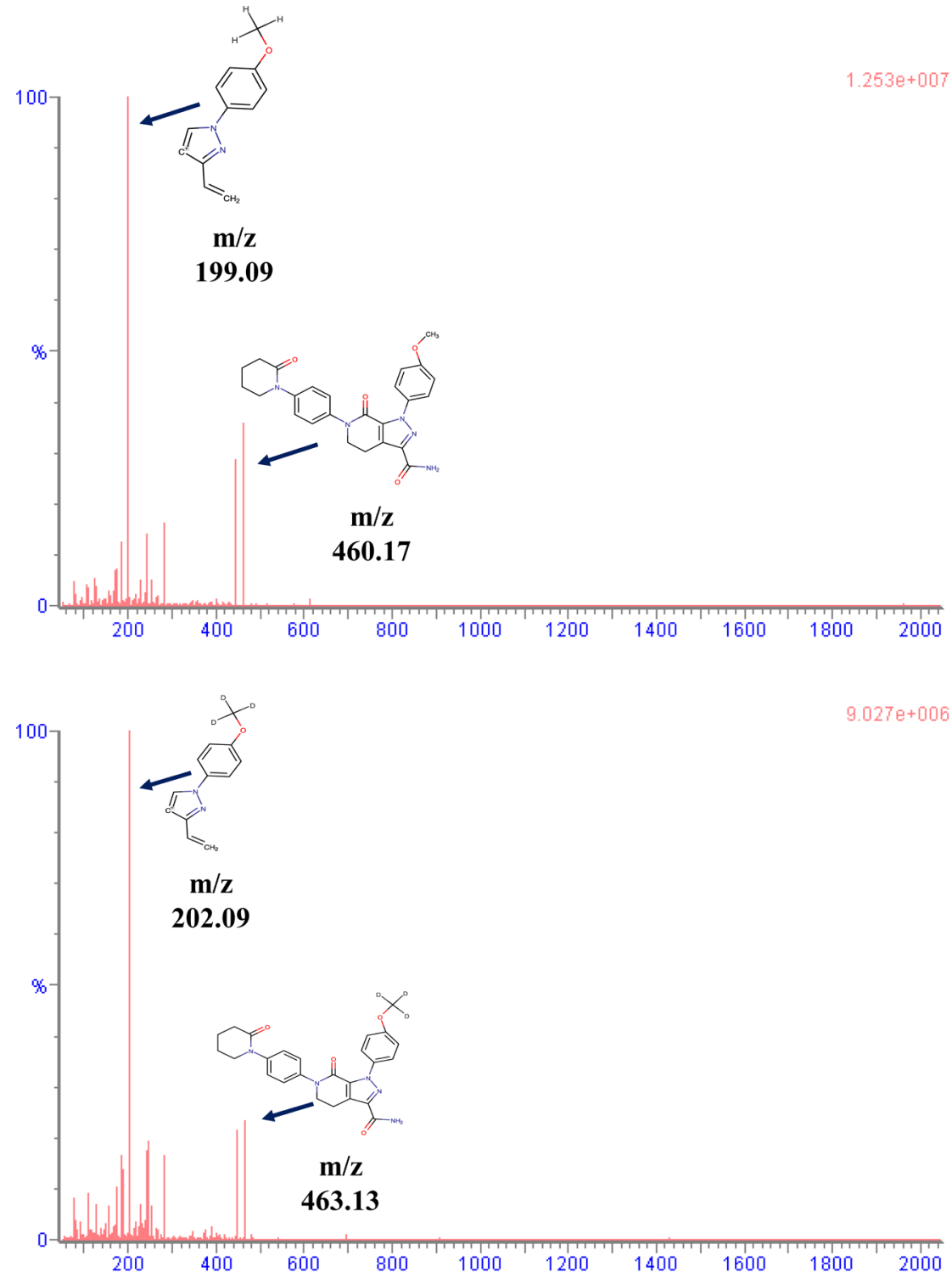

Figure 1. Mass spectra and chemical structures of (A) apixaban and (B) deuterated internal standard (apixaban-d3) in electrospray ionization (ESI) positive mode. The blue arrows indicate spectral peaks corresponding to each structure.

Table 1. Intra-batch and inter-batch accuracy and precision of validation batches

\begin{tabular}{|c|c|c|c|c|c|}
\hline \multicolumn{2}{|c|}{ Concentration (ng/mL) } & 1 & 2 & 15 & 400 \\
\hline \multirow{4}{*}{$\begin{array}{c}\text { Accuracy } \\
(\%)\end{array}$} & Batch 1 & 94.80 & 97.50 & 98.12 & 108.14 \\
\hline & Batch 2 & 106.40 & 97.80 & 97.55 & 104.51 \\
\hline & Batch 3 & 111.40 & 103.20 & 100.69 & 106.04 \\
\hline & Inter-batch & 104.20 & 99.50 & 98.79 & 106.23 \\
\hline \multirow{4}{*}{$\begin{array}{c}\text { Precision } \\
(\mathrm{CV}, \%)\end{array}$} & Batch 1 & 0.58 & 4.54 & 4.46 & 1.89 \\
\hline & Batch 2 & 4.49 & 4.52 & 1.41 & 2.13 \\
\hline & Batch 3 & 0.49 & 1.59 & 3.03 & 1.46 \\
\hline & Inter-batch & 8.72 & 3.31 & 2.24 & 2.24 \\
\hline
\end{tabular}


A
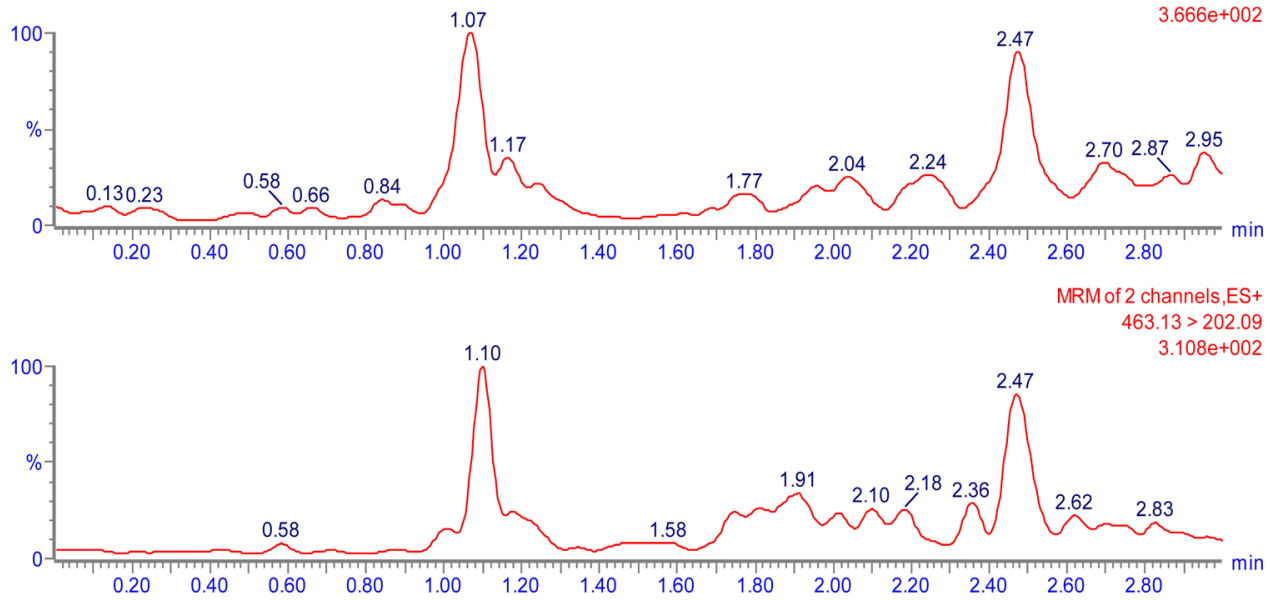

B

MRM of 2 channels, ES+ $460.17>199.09$
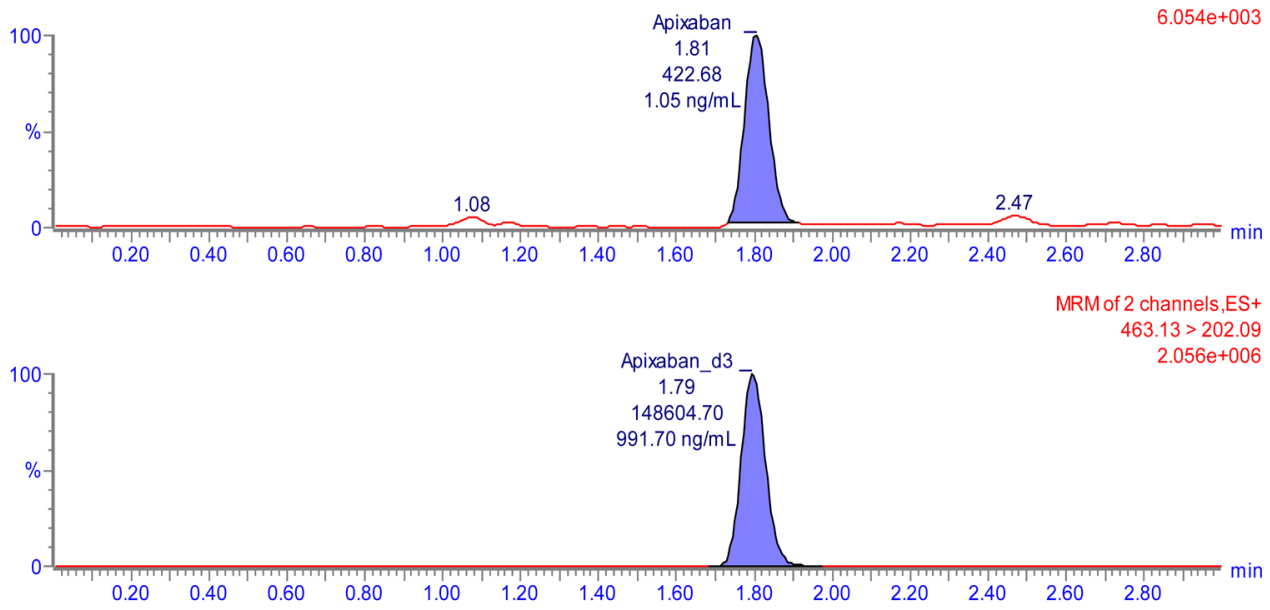

Figure 2. Representative chromatogram of (A) double blank human plasma and (B) lower limit of quantification samples.

A

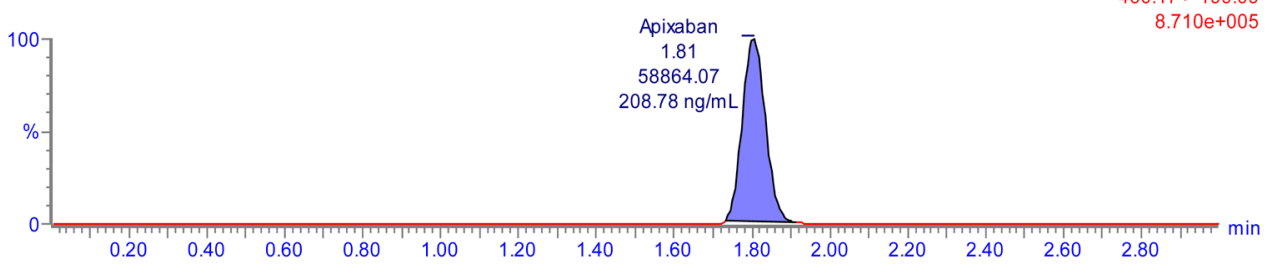

B

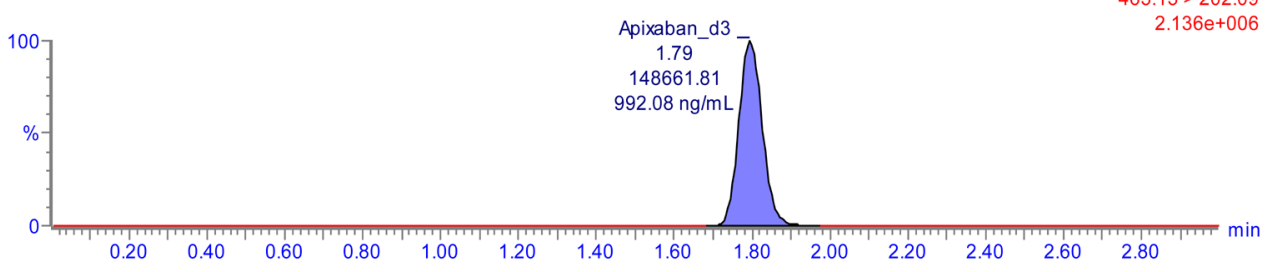

Figure 3. Representative chromatogram of $(A)$ apixaban and $(B)$ internal standard in plasma after oral administration of apixaban $10 \mathrm{mg}$. 
Table 2. Dilution integrity of apixaban in human blank plasma

\begin{tabular}{|c|c|c|c|c|c|c|}
\hline \multirow[b]{2}{*}{$N=5$} & \multicolumn{3}{|c|}{50 -fold dilution } & \multicolumn{3}{|c|}{ 10-fold dilution } \\
\hline & $\begin{array}{c}\text { Back-calculated } \\
\text { concentration (ng/mL) }\end{array}$ & Accuracy (\%) & Precision (\% CV) & $\begin{array}{c}\text { Back-calculated } \\
\text { concentration }(\mathrm{ng} / \mathrm{mL})\end{array}$ & Accuracy (\%) & Precision (\% CV) \\
\hline 1 & 1082.90 & 108.29 & \multirow{5}{*}{2.20} & 1043.50 & 104.37 & \multirow{5}{*}{2.43} \\
\hline 2 & 1037.40 & 103.74 & & 1037.50 & 103.77 & \\
\hline 3 & 1037.70 & 103.77 & & 1026.50 & 102.66 & \\
\hline 4 & 1028.10 & 102.81 & & 1086.50 & 108.67 & \\
\hline 5 & 1027.90 & 102.79 & & 1023.50 & 102.33 & \\
\hline Mean \pm SD & $1042.80 \pm 22.92$ & 104.28 & - & $1043.50 \pm 25.37$ & 104.36 & - \\
\hline
\end{tabular}

Table 3. Stability tests under various temperatures

\begin{tabular}{lcc}
\hline \multicolumn{1}{c}{ Concentration $(\mathrm{ng} / \mathrm{mL})^{\mathrm{c}}$} & 2 & 400 \\
\hline Re-inject $^{\mathrm{a}}$ & -0.66 & 0.60 \\
Processed sample $^{\mathrm{b}}$ & -0.34 & -0.21 \\
Freeze-thaw $^{\mathrm{c}}$ & -3.57 & -4.61 \\
Short-term $^{\mathrm{d}}$ & 3.74 & 2.42 \\
Stock short-term $^{\mathrm{e}}$ & -5.56 & 13.13 \\
\hline
\end{tabular}

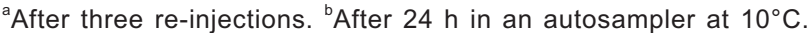
${ }^{\mathrm{c}}$ After three freeze-thaw cycles. ${ }^{\mathrm{d}} \mathrm{After} 20 \mathrm{~h}$ at room temperature. ${ }^{\mathrm{e}} \mathrm{After} 24 \mathrm{~h}$ at room temperature.

All of the results are presented as percentage (\%) change.

for apixaban at the LoQC and HiQC were $4.08 \%$ and $2.39 \%$, respectively. No significant matrix effect appeared in the human plasma with the LoQC and HiQC samples.

\section{Extraction efficiency and dilution integrity}

The extraction efficiency was evaluated at three QC concentrations, LoQC, MeQC, and HiQC, which showed recoveries of $16.57 \%, 15.24 \%$, and $14.66 \%$, respectively. The dilution integrity was assessed by diluting the DiQC $(1,000 \mathrm{ng} / \mathrm{mL})$ by 50 - and 10 -fold, respectively. The back-calculated nominal concentrations were 1042.8 and $1043.5 \mathrm{ng} / \mathrm{mL}$ for each sample. The accuracy and precision of the 50-fold dilution were 102.79-108.29\% and $2.20 \%$, respectively, and values for the 10 -fold dilution were $102.33-108.67 \%$ and $2.43 \%$, respectively. The results of the dilution integrity are presented in Table 2.

\section{Stability}

The stability of the apixaban working and stock solutions were evaluated under various conditions, and the results are presented in Table 3. The processed sample was stable for $24 \mathrm{~h}$ in the autosampler. The apixaban working and stock solutions were stable at laboratory temperatures for $10 \mathrm{~h}$. Six LoQC and HiQC samples were within $15 \%$ of the nominal concentration change measured after three repeated freeze-thaw cycles. The re-injection change was within $15 \%$ of the nominal concentration compared with the test and reference samples. The results indicated that the developed method could be used to analyze
A

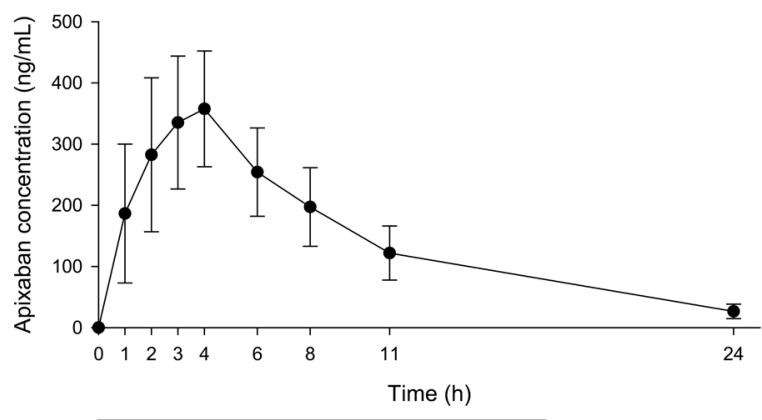

- $10 \mathrm{mg}$ apixaban administration $(n=23)$

B

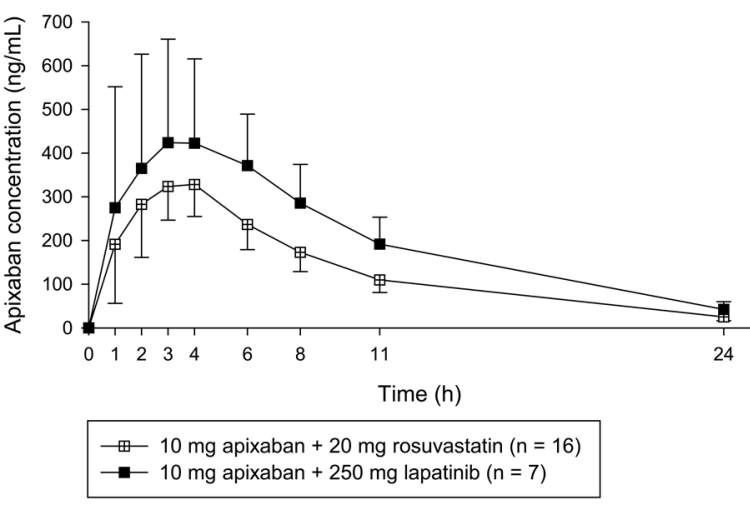

Figure 4. Mean time-concentration profile after $(A)$ administration of single dose of apixaban $10 \mathrm{mg}$ and (B) co-administration with $20 \mathrm{mg}$ rosuvastatin (crossed square) or $250 \mathrm{mg}$ lapatinib (solid square). Error bars represent the standard deviation (SD).

apixaban under the investigated conditions.

Application of the analysis method to a pharmacokinetic study The validated analytical method was applied to quantify apixaban in a clinical study. In Figure 4, the mean plasma apixaban concentration-time profiles for each group were presented. The mean \pm standard deviation (SD) values of the maximum plasma concentration $\left(\mathrm{C}_{\max }\right)$ and median (range) time to reach the $\mathrm{C}_{\max }\left(\mathrm{T}_{\max }\right)$ in the $10 \mathrm{mg}$ apixaban single administration group 
were $371.57 \pm 95.23 \mathrm{ng} / \mathrm{mL}$ and $4(1-4) \mathrm{h}$, respectively. The mean $\mathrm{C}_{\max }$ and median $\mathrm{T}_{\max }$ values of the $20 \mathrm{mg}$ rosuvastatin co-administered group were $347.65 \pm 73.63 \mathrm{ng} / \mathrm{mL}$ and $3(2-4)$ $\mathrm{h}$, respectively. The mean $\mathrm{C}_{\max }$ and median $\mathrm{T}_{\max }$ of the $250 \mathrm{mg}$ lapatinib co-administered group were $529.01 \pm 162.83 \mathrm{ng} / \mathrm{mL}$ and $3(1-6) \mathrm{h}$.

\section{Discussion}

An analytical method used for the measurement of apixaban concentration was developed and validated in this study. The percentage change in accuracy, precision, and stability under various temperature conditions in validation batches were within $15 \%$ of the nominal concentration at the LoQC, MeQC, and HiQC. Three different concentrations of QC samples did not show significant changes after repeated freeze-thaws for three cycles at $-80^{\circ} \mathrm{C}$, and no significant changes were observed compared with the nominal concentration even at laboratory and autosampler temperature conditions (10 and $24 \mathrm{~h}$, respectively). The time-concentration profiles for apixaban were determined using the developed method. Analyte ionization was performed in the ESI positive mode according to a previous report.[27,28] Comparing the positive and negative ionization mode, apixaban was detected in the positive ionization mode with high sensitivity. The mobile phases were evaluated using deionized water containing $0.1 \%$ formic acid, $2.5 \mathrm{mM}$ ammonium formate $(\mathrm{pH}$ 3.0), methanol containing $0.1 \%$ formic acid, and acetonitrile containing $0.1 \%$ formic acid using the Thermo Hypersil Gold C18 column. Among them, $2.5 \mathrm{mM}$ ammonium formate $(\mathrm{pH}$ 3.0) and methanol containing $0.1 \%$ formic acid (mobile phase $A$ and $\mathrm{B}$, respectively) showed the best peak shapes and excellent separation. Sample preparation using methanol showed better peak shape and reproducibility than those using acetonitrile did.

Generally, the chromogenic anti-factor Xa assay is used to quantify apixaban concentrations.[16,17] The correlation of apixaban concentrations and factor-Xa activity at the therapeutic range has been shown to be linear.[29-32] The dynamic ranges for apixaban were approximately 20-500 ng/mL.[17] This method was an indirect quantification method for apixaban using factor Xa activity with residual activated factor Xa. $[18,29]$ Changes in the coagulation factor or other anticoagulants may affect apixaban quantification.[21] Chromogenic assay agents are generally stable for up to 4 months depending on the storage temperature conditions after reconstitution.[33] For example, STA ${ }^{\oplus}$-Liquid Anti-Xa (Stago Group, France) is stable for 3 months at $2-8^{\circ} \mathrm{C}$ and Technochrom ${ }^{\oplus}$ anti-Xa (DiaPharma Group, Inc., West Chester Township, OH, USA) is stable for 4 months at $2-8^{\circ} \mathrm{C}$. The direct quantification of apixaban using LC-MS could solve these drawbacks, as the analysis with this technique has high sensitivity and specificity.[34] In addition, relatively flexible ranges of the calibration curve could be used according to the study specification or sample status. Chromogenic or clotting-based assays such as prothrombin time (PT), thrombin time (TT), the chromogenic factor-Xa assay, and other methods indirectly quantify coagulation activity,[21] whereas the analytical method using LC-MS enables the direct quantification of anticoagulant concentrations.[21] The other advantage of using LC-MS is that it is not affected by changes in other anticoagulants or coagulation factors. In previously reported studies, apixaban was shown to be stable at $-50^{\circ} \mathrm{C}$ and $-80^{\circ} \mathrm{C}$ for 6 and 8 months, respectively. [27,32] Therefore, the analysis method using LC-MS may be useful for apixaban analysis that requires high selectivity and sensitivity such as for pharmacokinetic studies. Compared with previously reported methods, $[12,21,28,35,36]$ the concentration of apixaban analyzed within 3 min on a C18 column with reliable reproducibility (accuracy and precision were within 15\% range). Sample preparation with protein precipitation (PP) was used in the current study. PP is a simple and rapid preparation method compared with solid-phase extraction (SPE) or liquid-liquid extraction (LLE).[37,38] As the current method was rapid and relatively simple, it might be suitable for therapeutic monitoring or large scale sample analysis. A limitation of this study was that the method showed lower extraction efficiency than that of previous studies.[21,39] The three-replicated mean extraction efficiencies were calculated to be $16.57 \%, 15.24 \%$, and $14.66 \%$ for the LoQC, MeQC, and HiQC, respectively. In the other reported studies, the extraction efficiencies were 104\% [21] and ranged from $101.8 \%$ to $102.7 \%$.[39] The extraction efficiency range in this study was $82 \%$ to $84 \%$ when it was calculated using the same method as that used in previous studies.[21,39] Although the extraction efficiency in this study was relatively lower than that previously reported, the relative SD (RSD) of the mean extraction efficiency was $6.5 \%$ (within 15\%), indicating the reproducibility of the validation method; therefore, this method was considered reliable.

The $\mathrm{C}_{\max }$ and $\mathrm{T}_{\max }$ values were compared with other previous studies. In this study, the arithmetic mean and geometric mean \pm standard deviation (SD) of $\mathrm{C}_{\max }$ were $371.57 \pm 95.23 \mathrm{ng} / \mathrm{mL}$ and $358.50 \pm 1.32 \mathrm{ng} / \mathrm{mL}$, respectively. Median $\mathrm{T}_{\max }$ (range) was 4 (1-4) h. Frost, C. et al. reported the arithmetic mean \pm SD of $\mathrm{C}_{\max }$ and median (range) $\mathrm{T}_{\max }$ were $176.3 \pm 42 \mathrm{ng} / \mathrm{mL}$ and 3.0 (2.5-4.0) $\mathrm{h}$ after the single administration of apixaban $10 \mathrm{mg}$ in healthy White and Black volunteers.[40] Upreti, V. V. et al. reported the geometric mean $\pm \mathrm{SD}$ of $\mathrm{C}_{\max }$ and median $\mathrm{T}_{\max }$ (range) were $207 \pm 24 \mathrm{ng} / \mathrm{mL}$ and 3.03 (2.0-6.0) h after single administration of apixaban $10 \mathrm{mg}$ in healthy White, Black and Asian volunteers. [41] Moreover Cui, Y. et al. reported the geometric mean $\mathrm{C}_{\max }(\mathrm{CV})$ and median $\mathrm{T}_{\max }$ (min, max) were 233.9 (26) $\mathrm{ng} / \mathrm{mL}$ and $3(2,4) \mathrm{h}$ after the same dosage administration in healthy Chinese volunteers.[42] $\mathrm{The}_{\mathrm{T}} \mathrm{T}_{\max }$ was comparable with other reports, whereas the $\mathrm{C}_{\max }$ was higher than the compared results. Apixaban was known as primarily metabolized by CYP3A4 and is a substrate of P-gp and BCRP. Genetic polymorphism, such as the ABCB1, ABCB2 and CYP3A5 gene, affected the disposition of apixaban. $[43,44]$ Further studies with phar- 
macogenetics approach might be needed to identify the cause of the differences of apixaban pharmacokinetic parameters in a different population.

\section{Conclusions}

An analytical method for the determination of apixaban was developed using UPLC-MS/MS. Previous anti-factor Xa assays indirectly measured the concentrations of apixaban. These methods were affected by changes in coagulant factors or coadministered drugs. However, the developed method using LCMS in this study could be directly used to quantify the concentration of apixaban and had an LLOQ value of $1 \mathrm{ng} / \mathrm{mL}$, which was lower than that of the anti-factor Xa assay method (approximately $20 \mathrm{ng} / \mathrm{mL}$ ). Therefore, the LC-MS-based analytical method may be useful for pharmacokinetic studies and clinical applications.

\section{Acknowledgments}

This research was supported by the Bio \& Medical Technology Development Program of the National Research Foundation (NRF) and was funded by the Korean government (MSIP\&MOHW) (No. NRF-2015M3A9E1028327).

\section{Conflict of interest}

- Authors: All authors declare no competing financial interests.

- Reviewers: Nothing to declare

- Editors: Nothing to declare

\section{References}

1. Hart RG, Pearce LA, Aguilar MI. Meta-analysis: antithrombotic therapy to prevent stroke in patients who have nonvalvular atrial fibrillation. Ann Intern Med 2007;146:857-867.

2. Wolf PA, Abbott RD, Kannel WB. Atrial fibrillation as an independent risk factor for stroke: the Framingham Study. Stroke 1991;22:983-988.

3. Favaloro EJ, Lippi G. Laboratory testing in the era of direct or non-vitamin $\mathrm{K}$ antagonist oral anticoagulants: a practical guide to measuring their activity and avoiding diagnostic errors. Semin Thromb Hemost 2015;41:208227. doi: 10.1055/s-0035-1546827.

4. Gage BF, Finn SD, White RH. Management and dosing of warfarin therapy. Am J Med 2000;109:481-488. doi: https://doi.org/10.1016/S00029343(00)00545-3.

5. Granger CB, Alexander JH, McMurray JJ, Lopes RD, Hylek EM, Hanna M, et al. Apixaban versus Warfarin in Patients with Atrial Fibrillation. N Engl J Med 2011;365:981-992. doi: 10.1056/NEJMoa1107039.

6. Conway SE, Hwang AY, Ponte CD, Gums JG. Laboratory and clinical monitoring of direct acting oral anticoagulants: what clinicians need to know. Pharmacotherapy 2017;37:236-248. doi: 10.1002/phar.1884.

7. Johnson JA, Gong L, Whirl-Carrillo M, Gage BF, Scott SA, Stein CM, et al. Clinical Pharmacogenetics Implementation Consortium Guidelines for CYP2C9 and VKORC1 genotypes and warfarin dosing. Clin Pharmacol Ther 2011;90:625-629. doi: 10.1038/clpt.2011.185.

8. Ansell J, Hirsh J, Hylek E, Jacobson A, Crowther M, Palareti G. Pharmacology and Management of the Vitamin K Antagonists: American College of Chest Physicians Evidence-Based Clinical Practice Guidelines (8th Edition). Chest 2008;133:160S-198S. doi: 10.1378/chest.08-0670.

9. Ufer M. Comparative pharmacokinetics of vitamin K antagonists: warfarin, phenprocoumon and acenocoumarol. Clin Pharmacokinet 2005;44:12271246. doi: 10.2165/00003088-200544120-00003.

10. Wells PS, Holbrook AM, Crowther NR, Hirsh J. Interactions of warfarin with drugs and food. Ann Intern Med 1994;121:676-683.
11. Harder S, Graff J. Novel oral anticoagulants: clinical pharmacology, indications and practical considerations. Eur J Clin Pharmacol 2013;69:16171633. doi: 10.1007/s00228-013-1510-z.

12. Gous T, Couchman L, Patel JP, Paradzai C, Arya R, Flanagan RJ. Measurement of the direct oral anticoagulants apixaban, dabigatran, edoxaban, and rivaroxaban in human plasma using turbulent flow liquid chromatography with high-resolution mass spectrometry. Ther Drug Monit 2014;36:597-605. doi: 10.1097/FTD.0000000000000059.

13. Wong PC, Pinto DJ, Zhang D. Preclinical discovery of apixaban, a direct and orally bioavailable factor $\mathrm{Xa}$ inhibitor. J Thromb Thrombolysis 2011;31:478-492. doi: 10.1007/s11239-011-0551-3.

14. Weitz Jl. Meeting the unmet needs in anticoagulant therapy. Eur $\mathrm{J}$ Haematol Suppl 2010:1-28. doi: 10.1111/j.1600-0609.2010.01461.x.

15. Song Y, Chang M, Suzuki A, Frost RJ, Kelly A, LaCreta F, et al. Evaluation of Crushed Tablet for Oral Administration and the Effect of Food on Apixaban Pharmacokinetics in Healthy Adults. Clin Ther 2016;38:1674-1685. e1671. doi: https://doi.org/10.1016/j.clinthera.2016.05.004.

16. Hillarp A, Gustafsson KM, Faxälv L, Strandberg K, Baghaei F, Blixter IF, et al. Effects of the oral, direct factor Xa inhibitor apixaban on routine coagulation assays and anti-FXa assays. J Thromb Haemost 2014;12:15451553. doi: 10.1111/jth.12649.

17. Beyer J, Trujillo T, Fisher S, Ko A, Lind SE, Kiser TH. Evaluation of a Heparin-Calibrated Antifactor Xa Assay for Measuring the Anticoagulant Effect of Oral Direct Xa Inhibitors. Clin Appl Thromb Hemost 2016;22:423428. doi: 10.1177/1076029616629759.

18. Douxfils J, Pochet L, Lessire S, Vancraeynest C, Dogné J-M, Mullier F. Mass spectrometry in the therapeutic drug monitoring of direct oral anticoagulants. Useful or useless? TrAC Trends Analyt Chem 2016;84:41-50. doi: https://doi.org/10.1016/j.trac.2016.01.029.

19. Lim MS, Chapman K, Swanepoel P, Enjeti AK. Sensitivity of routine coagulation assays to direct oral anticoagulants: patient samples versus commercial drug-specific calibrators. Pathology 2016;48:712-719. doi: https://doi.org/10.1016/j.pathol.2016.07.008.

20. Harenberg J, Kraemer S, Du S, Giese C, Schulze A, Kraemer R, et al. Determination of direct oral anticoagulants from human serum samples. Semin Thromb Hemost 2014;40:129-134. doi: http://dx.doi.org/10.1055/ s-0033-1363462.

21. Schmitz EM, Boonen K, van den Heuvel DJ, van Dongen JL, Schellings MW, Emmen JM, et al. Determination of dabigatran, rivaroxaban and apixaban by ultra-performance liquid chromatography - tandem mass spectrometry (UPLC-MS/MS) and coagulation assays for therapy monitoring of novel direct oral anticoagulants. J Thromb Haemost 2014;12: 1636-1646. doi: 10.1111/jth.12702.

22. Saint-Marcoux F, Sauvage FL, Marquet P. Current role of LC-MS in therapeutic drug monitoring. Anal Bioanal Ch 2007;388:1327-1349.

23. Lim CK, Lord G. Current developments in LC-MS for pharmaceutical analysis. Biol Pharm Bull 2002;25:547-557.

24. Baldelli S, Cattaneo D, Pignatelli P, Perrone V, Pastori D, Radice S, et al. Validation of an LC-MS/MS method for the simultaneous quantification of dabigatran, rivaroxaban and apixaban in human plasma. Bioanalysis 2016;8:275-283. doi: 10.4155/bio.15.261.

25. Guidance for Industry - Bioanalytical Method Validation (2013) FDA https://www.fda.gov/downloads/drugs/guidances/ucm368107.pdf. Accessed 21 June 2018

26. Guideline on bioanalytical method validation (2013) Korea Ministry of Food and Drug Safety. http://nifds.go.kr/_custom/nifds/_common/board/ download.jsp?attach_no=18470. Accessed 21 June 2018

27. Baig MLA, Ali SA. A Validated LC-MS/MS Method for the Estimation of Apixaban in Human Plasma. J App Pharm Sci 2017;7:044-052.

28. Zhang WL, Lou D, Zhang DT, Zhang Y, Huang HJ. Determination of rivaroxaban, apixaban and edoxaban in rat plasma by UPLC-MS/MS method. J Thromb Thrombolysis 2016;42:205-211. doi: 10.1007/s11239-0161367-y.

29. Douxfils J, Chatelain C, Chatelain B, Dogné JM, Mullier F. Impact of apixaban on routine and specific coagulation assays: a practical laboratory guide. Thromb Haemost 2013;110:283-294. doi: 10.1160/TH12-12-0898.

30. Shin H, Cho MC, Kim RB, Kim CH, Choi NC, Kim SK, et al. Laboratory measurement of apixaban using anti-factor $\mathrm{Xa}$ assays in acute ischemic stroke patients with non-valvular atrial fibrillation. J Thromb Thrombolysis 
2018;45:250-256. doi: 10.1007/s11239-017-1590-1.

31. Gouin-Thibault I, Flaujac C, Delavenne X, Quenet S, Horellou MH, Laporte S, et al. Assessment of apixaban plasma levels by laboratory tests: suitability of three anti-Xa assays. Thromb Haemo 2014;112:240-248. doi: 10.1160/TH13-06-0470.

32. Derogis PB, Sanches LR, de Aranda VF, Colombini MP, Mangueira CL, Katz $M$, et al. Determination of rivaroxaban in patient's plasma samples by anti-Xa chromogenic test associated to High Performance Liquid Chromatography tandem Mass Spectrometry (HPLC-MS/MS). PLoS One 2017;12:e0171272. doi: 10.1371/journal.pone.0171272.

33. Newall F. Anti-factor Xa (Anti-Xa) Assay. In: Monagle P (ed) Haemostasis: Methods and Protocols. Humana Press, Totowa, NJ, 2013;265-272. doi:10.1007/978-1-62703-339-8_19

34. Vogeser M, Seger C. A decade of HPLC-MS/MS in the routine clinical laboratory - Goals for further developments. Clin Biochem 2008;41:649662. doi: 10.1016/j.clinbiochem.2008.02.017.

35. Skeppholm M, Al-Aieshy F, Berndtsson M, Al-Khalili F, Rönquist-Nii Y, Söderblom $\mathrm{L}$, et al. Clinical evaluation of laboratory methods to monitor apixaban treatment in patients with atrial fibrillation. Thromb Res 2015;136:148-153. doi: https://doi.org/10.1016/j.thromres.2015.04.030.

36. Pursley J, Shen JX, Schuster A, Dang OT, Lehman J, Buonarati MH, et al. LC-MS/MS determination of apixaban (BMS-562247) and its major metabolite in human plasma: an application of polarity switching and monolithic HPLC column. Bioanalysis 2014;6:2071-2082. doi: 10.4155/bio.14.66.

37. Ashri NY, Abdel-Rehim M. Sample treatment based on extraction techniques in biological matrices. Bioanalysis 2011;3:2003-2018. doi: 10.4155/ bio.11.201.
38. Kole PL, Venkatesh G, Kotecha J, Sheshala R. Recent advances in sample preparation techniques for effective bioanalytical methods. Biomed Chromatogr 2011;25:199-217. doi: 10.1002/bmc.1560.

39. Wiesen MH, Blaich C, Streichert T, Michels G, Müller C. Paramagnetic micro-particles as a tool for rapid quantification of apixaban, dabigatran, edoxaban and rivaroxaban in human plasma by UHPLC-MS/MS. Clin Chem Lab Med 2017;55:1349-1359. doi: 10.1515/cclm-2016-0888.

40. Frost C, Wang J, Nepal S, Schuster A, Barrett YC, Mosqueda-Garcia R, et al. Apixaban, an oral, direct factor $\mathrm{Xa}$ inhibitor: single dose safety, pharmacokinetics, pharmacodynamics and food effect in healthy subjects. $\mathrm{Br} \mathrm{J}$ Clin Pharmacol 2013;75:476-487. doi: 10.1111/j.1365-2125.2012.04369.x.

41. Upreti VV, Wang J, Barrett YC, Byon W, Boyd RA, Pursley J, et al. Effect of extremes of body weight on the pharmacokinetics, pharmacodynamics, safety and tolerability of apixaban in healthy subjects. Br J Clin Pharmacol 2013;76:908-916. doi: 10.1111/bcp.12114.

42. Cui Y, Song Y, Wang J, Yu Z, Schuster A, Barrett YC, et al. Single-and multiple-dose pharmacokinetics, pharmacodynamics, and safety of apixaban in healthy Chinese subjects. Clin Pharmacol 2013;5:177-184. doi: 10.2147/CPAA.S51981.

43. Dimatteo C, D'Andrea G, Vecchione G, Paoletti O, Tiscia GL, Santacroce R, et al. ABCB1 SNP rs4148738 modulation of apixaban interindividual variability. Thromb Res 2016;145:24-26 doi: 10.1016/j.thromres.2016.07.005.

44. Ueshima S, Hira D, Fujii R, Kimura Y, Tomitsuka C, Yamane T, et al. Impact of ABCB1, ABCG2, and CYP3A5 polymorphisms on plasma trough concentrations of apixaban in Japanese patients with atrial fibrillation. Pharmacogenet Genomics 2017;27:329-336. doi: 10.1097/FPC.0000000000000294. 